\title{
Oscillation of Nonlinear Vector Differential Equations (*).
}

E. S. NoussalR and C. A. Swanson (Vancouver, Canada and Sydney, Australia)

Summary. - Oscillation criteria are obtained for vector partial differential equations of the type $\Delta v+b(x, v) v=0, x \in G, v \in E^{m}$, where $G$ is an exterior domain in $E^{n}$, and $b$ is a continuous nonnegative valued function in $G \times E^{m}$. A solution $v: G \rightarrow E^{m}$ is called h-oscillatory in $G$ whenever the scalar product $[v(x), h](|h|=1)$ has zeros $x$ in $G$ with $|x|$ arbitrarily large. It is shown that the spherical mean of $[v(x), h]$ over a hypersphere of radius $r$ in $E^{n}$ satisfies a nonlinear ordinary differential inequality. As a consequence, the main theorems give suffieient conditions on $b(x, t)$, depending upon the dimension $n$, for all solutions $v$ to be h-oscillatory in $G$.

Let $G$ be an exterior domain in $n$-dimensional Euclidean space $E^{n}$, i.e. suppose that $G$ contains the complement of some $n$-ball in $E^{n}$. Points in $E^{n}$ will be denoted by $x=\left(x_{1}, \ldots, x_{n}\right)$ and the Euclidean length of $x$ by $|x|$. We shall consider $m$-vector functions $v: G \rightarrow E^{m}$ whose components satisfy a system of nonlinear partial differential equations in $G$. The notation $[v(x), w(x)]$ will be used for the scalar product of $v(x), w(x) \in E^{m}$ and $|v(x)|$ for the Euclidean length of $v(x)$. Our objective is to obtain oscillation criteria for the vector differential operator defined by

$$
L v \equiv \Delta v+b(x, v) v=0, \quad x \in G
$$

where

$$
\Delta=\sum_{i=1}^{n} D_{i} D_{i}, \quad D_{i}=\partial / \partial x_{i}, \quad i=1, \ldots, n
$$

The following assumptions on the function $b$ will be retained in the sequel.

\section{(2) Assumptions}

$b: G \times E^{m} \rightarrow E^{1}$ is a continuous nonnegative valued function such that

$$
b(x, v) \geqslant \lambda(|x|) \psi(|v|)
$$

for all $x \in G, v \in E^{m}$, where

(i) $\lambda$ is a nonnegative continuous function in $[0, \infty)$;

(ii) $\psi \in O^{1}[0, \infty)$ with $\psi(t)>0$ for $t \neq 0$ and $\psi^{\prime}(t) \geqslant 0$ for all $t$; and

(iii) The function $\varphi$ defined by $\varphi(t)=t \psi(t)$ is convex in $[0, \infty)$.

(*) Entrata in Redazione il 26 giugno 1975. 
The domain $\mathfrak{D}_{L}$ of $L$ is defined to be the set of all $m$-vector functions $v: G \rightarrow E^{m}$ with real components $v_{k} \in C^{2}(G), k=1, \ldots, m$.

The following notation will be used:

$$
\begin{aligned}
& S_{a}=\left\{x \in E^{n}:|x|>a\right\} ; \\
& G_{a}=G \cap S_{a} ; \\
& \Omega_{a}=\left\{x \in E^{n}:|x|=a\right\}, \quad a>0 .
\end{aligned}
$$

Our assumption that $G$ is an exterior domain means that $S_{a} \subset G$ for some $a>0$. The definition of oscillation of $L$ in a direction $h$ (called $h$-oscillation) given below has been used by the authors [2] and by Ju. I. DomšLAK [1] in special cases.

Definition. - A function $v: G \rightarrow E^{m}$ is called $h$-ose in $G \subset E^{n}$ iff $[v(x), h]$ has a zero in $G_{a}$ for all $a>0$, where $h$ is a constant unit vector in $E^{m}$. The differential operator $L$ is called $h$-ose in $G$ whenever every solution $v \in \mathfrak{D}_{L}$ of $L v=0$ is $h$-ose in $G$.

This definition means that the projection of an $h$-ose function $v$ on $h$ is oscillatory in $G$ in the classical sense, i.e. this projection has zeros $x \in G$ with $|x|$ arbitrarily large. Examples are given in [2] to show that the $h$-ose property of an operator $L$ might depend upon $h$, e.g. it might be satisfied for $h^{*}=(1,0)$ but not for $h^{*}=(0,1)$.

An example of a function $b: G \times E^{m} \rightarrow E^{1}$ for which assumptions (2) are satisfied is

$$
b(x, v)=g^{*}(v) A(x) g(v), \quad x \in G,
$$

where $A(x)$ is a symmetric, uniformly positive definite, continuous matrix function, and $g$ is a continuous $m$-vector valued function such that

$$
|g(v)|^{2} \geqslant \psi(|v|)
$$

with $\psi$ satisfying assumptions (2ii) and (2iii). Then $b(x, v) \geqslant \lambda(r) \psi(|v|)$ for all $x \in G$, $v \in E^{m}$, where $\lambda(r)$ denotes the minimum of the smallest eigenvalue of $A(x)$ on the hypersphere $\Omega_{r}$. Clearly this example provides a fairly wide class of nonlinear systems (1) for which all the assumptions (2) are satisfied. An Emden-Fowler prototype is the case $g(v)=|v|^{\alpha} v, \alpha \geqslant-1$. A slightly different example is

$$
b(x, v)=\left[v^{*} A(x) v\right]^{\gamma}, \quad x \in G
$$

with $A(x)$ as before and $\gamma \geqslant 0$.

The $h$-oscillation theorems given by the authors in [2] do not apply to the above nonlinear examples since the majorization hypothesis given there is not fulfilled. On the other hand, we do not consider the case that $b$ is matrix-valued in the present 
paper. Unlike the theorems in [2], our results are primarily nonlinear. For completeness, however, we state Theorem 3 in such a way that it is applicable to both the linear and nonlinear cases.

The spherical mean-projection of an $L^{1} m$-vector function $v: G \rightarrow E^{m}$ over $\Omega_{r}$, $r>a$, is defined as

$$
f(r)=f_{h}(r ; v)=\frac{1}{s\left(\Omega_{r}\right)} \int_{\Omega_{r}}[v(x), h] d s,
$$

where $s$ denotes the measure on $\Omega_{r}$.

LEMma. - Under assumptions (2), let $v \in \mathscr{D}_{L}$ be a solution of (1) such that $[v(x), h]$ has constant sign on $\Omega_{r}$, where $h$ is a constant unit $m$-vector. Then the spherical mean-projection (3) of $v$ over $\Omega_{r}$ satisfies the ordinary differential inequality

$$
\left[r^{n-1}|f(r)|^{\prime}\right]^{\prime}+r^{n-1} \lambda(r) \varphi[|f(r)|] \leqslant 0
$$

for $a<r<\infty$.

Proof. - First consider the case that $[v(x), h]>0$ on $\Omega_{r}$. Since $[v(x), h] \leqslant$ $\leqslant|v(x)||h|=|v(x)|$ and $\psi$ is a nondecreasing function by (2ii), we find from (1) that

$$
\begin{aligned}
\Delta[v, h] & =-b(x, v)[v, h] \\
& \leqslant-\lambda(r) \psi(|v|)[v, h] \\
& \leqslant-\lambda(r) \psi([v, h])[v, h],
\end{aligned}
$$

where $r=|x|$. For $[v(x), h]<0$ we have similarly

$$
\Delta[v, h] \geqslant-\lambda(r) \psi(|[v, h]|)[v, h] .
$$

It follows from (5) and (6) that $u=|[v, h]|$ satisfies the differential inequality

$$
\Delta u \leqslant-\lambda(r) u \psi(u)=-\lambda(r) \varphi(u)
$$

The definition (3) reduces to

$$
|f(r)|=\frac{r^{1-n}}{\omega_{n}} \int_{\Omega_{r}} u(x) d s,
$$

where $\omega_{n}$ denotes the measure of the unit $(n-1)$-sphere in $E^{n}$. Thus

$$
|f(r)|=\frac{r^{1-n}}{\omega_{n}} \int_{\Omega_{r}} u(x) \sum_{i=1}^{n} \frac{x_{i}^{2}}{r^{2}} d s, \quad r=|x| .
$$


A formula which can be used to differentiate such an integral with respect to $r$ has been given by the authors [3]:

$$
\begin{gathered}
\frac{d}{d r} \int_{\Omega_{r}} w(x) \frac{x_{i}}{r} d s=\int_{\Omega_{r}} D_{i} w(x) d s, \\
D_{i}=\partial / \partial x_{i}, i=1, \ldots, n .
\end{gathered}
$$

We apply (9) to (8) to obtain

$$
\begin{aligned}
\omega_{n} r^{r-1}|f(r)|^{\prime} & =\int_{\Omega_{r}} \sum_{i} D_{i}\left(\frac{u(x) x_{i}}{r}\right) d s+\frac{1-n}{r} \int_{\Omega_{r}} u(x) d s \\
& =\int_{\Omega_{r}}\left(\sum_{i} D_{i} u\right) \frac{x_{i}}{r} d s
\end{aligned}
$$

since $\sum D_{i}\left(x_{i} / r\right)=(n-1) / r$. Applying (9) again we find that

$$
\begin{aligned}
\omega_{n}\left[r^{n-1}|f(r)|^{\prime}\right]^{\prime} & =\int_{\Omega_{r}} \sum D_{i}^{2} u d s \\
& =\int_{\Omega_{r}} \Delta u d s \\
& \leqslant-\lambda(r) \int_{\Omega_{r}} \varphi(u(x)) d s
\end{aligned}
$$

on account of (7). From Jensen's inequality for convex functions $\varphi[4$, p. 160],

$$
\varphi\left[\frac{1}{s\left(\Omega_{r}\right)} \int_{\Omega_{r}} u(x) d s\right] \leqslant \frac{1}{s\left(\Omega_{r}\right)} \int_{\Omega_{r}} \varphi[u(x)] d s .
$$

Then (10) implies the differential inequality

$$
\begin{aligned}
{\left[r^{n-1}|f(r)|^{\prime}\right]^{\prime} } & \leqslant-\frac{\lambda(r)}{\omega_{n}} s\left(\Omega_{r}\right) \varphi[|f(r)|] \\
& =-r^{n-1} \lambda(r) \varphi[|f(r)|] .
\end{aligned}
$$

This completes the proof of the Lemma.

On the basis of this Lemma, $h$-oscillation theorems for (1) will follow from appropriate oscillation criteria for the ordinary differential inequality (4). Theorems $A$ and $B$ below apply to the differential inequality

$$
\left[a(r) y^{\prime}(r)\right]^{\prime}+b(r) \varphi[y(r)] \leqslant 0
$$


under the following hypotheses

$$
\begin{aligned}
& \text { (A) } a \in C^{\mathrm{I}}[0, \infty) \text { with } a(r)>0 \text { in }[0, \infty) \text {; } \\
& \text { (B) } b \in C[0, \infty) \text { with } b(r) \geqslant 0 \text { in }[0, \infty) \text {; } \\
& \text { (C) } \varphi \in C^{1}[0, \infty), \varphi(t)>0 \text { for } t \neq 0, \\
& \text { and } \varphi^{\prime}(t) \geqslant 0 \text { in }(0, \infty) .
\end{aligned}
$$

Evidently (4) is the special case of (11) for which

$$
a(r)=r^{n-1}, \quad b(r)=r^{n-1} \lambda(r),
$$

and hypotheses (12) are all fulfilled on account of (2).

THEOREM A. - Inequality (11) has no solution $y$ which is positive throughout $\left[r_{0}, \infty\right)$ for any $r_{0}>0$ if $a, b$, and $\varphi$ satisfy hypotheses (12) and the following conditions

$$
\begin{aligned}
& \text { (i) } \int_{a}^{\infty} \frac{d r}{a(r)}=\infty ; \\
& \text { (ii) } \int_{e}^{\infty}\left(\int_{c}^{r} \frac{d s}{a(s)}\right) b(r) d r=\infty \text {; and } \\
& \text { (iii) } \int_{0}^{\infty} \frac{d t}{\varphi(t)}<\infty
\end{aligned}
$$

for some $c>0$.

In the event that condition (i) fails, we need the additional hypothesis that the function $\varphi$ in (11) satisfies

$$
\varphi(s t) \geqslant \varphi_{0}(s) \varphi_{0}(t)
$$

for all $s>0, t>0$, where $\varphi_{0}$ is a function satisfying assumption (12C).

ThEorem B. - Inequality (11) has no solution $y$ which is positive throughout $\left[r_{0}, \infty\right)$ for any $r_{0}>0$ if $a, b$, and $\varphi$ satisfy (12), (13), and the following conditions

$$
\text { (i) } \int_{e}^{\infty} \frac{d r}{a(r)}<\infty \text {; }
$$




$$
\begin{aligned}
& \text { (ii) } \int_{c}^{\infty} \varphi_{0}\left(\int_{r}^{\infty} \frac{d s}{a(s)}\right) b(r) d r=\infty \text {; and } \\
& \text { (iii) } \int_{e}^{\infty} \frac{d t}{\varphi_{0}(t)}<\infty
\end{aligned}
$$

for some $e>0$.

Proofs of Theorems A and B are given in the appendix.

Theorem 1. - The differential operator $L$ given by (1) is $h$-ose in an exterior domain $G$ of $E^{2}$ if assumptions (2) hold and

$$
\begin{aligned}
& \text { (i) } \int^{\infty} r \log r \lambda(r) d r=\infty ; \\
& \text { (ii) } \int^{\infty} \frac{d u}{\varphi(u)}<\infty .
\end{aligned}
$$

Proof. - Suppose to the contrary that $v$ is a solution of (1) such that $[v(x), h]$ has a fixed sign in $G_{r}$ for all $r>R$. If $[v(x), h]>0$ in $G_{r}$ for $r>R$, then $[v(x), h]>0$ on $\Omega_{r}$ for $r>r_{0}$, where $r_{0}=\max (a, R)$. Hence $f(r)>0$ for $r>r_{0}$ (see (3)) and $f(r)$ satisfies (4) by the Lemma. This contradicts Theorem $A$ in the case

$$
a(r)=r, \quad b(r)=r \lambda(r)
$$

according to hypotheses (i) and (ii). If $[v(x), h]<0$ in $G_{r}$ for all $r>R$, then $|f(r)|$ satisfies (4), and a similar contradiction is obtained.

Theorem 2. - The differential operator $L$ is $h$-ose in an exterior domain $G$ of $E^{n}$ $(n \geqslant 3)$ if (2) and (13) hold, and

$$
\begin{aligned}
& \text { (i) } \int^{\infty} \varphi_{0}\left(\frac{r^{2-n}}{n-2}\right) r^{n-1} \lambda(r) d r=\infty ; \\
& \text { (ii) } \int^{\infty} \frac{d u}{\varphi_{0}(u)}<\infty .
\end{aligned}
$$

This follows from Theorem $B$ in the case

$$
a(r)=r^{n-1}, \quad b(r)=r^{n-1} \lambda(r), \quad n \geqslant 3
$$

similarly to Theorem 1. 
Theorems 1 and 2 exclude the linear case $\varphi(u)=u$ because of the hypothesis (ii) of either theorem. In order to obtain similar (but slightly weaker) results applicable to both linear and nonlinear operators $L$, we employ the following replacement for condition (13): For arbitrary $\delta>0$ there exists a corresponding $K(\delta)>0$ and a number $\gamma \geqslant 1$ such that

$$
\frac{u^{\gamma}}{\varphi(u)} \leqslant \Pi(\delta)
$$

for all $u \geqslant \delta$. Condition (14) holds, for example, when $\varphi$ has the form

$$
\varphi(u)=u^{y}[\log (|u|+1)]^{\beta}, \quad \gamma \geqslant 1, \beta \geqslant 0
$$

including the linear case $(\gamma=1, \beta=0)$ and the superlinear case $(\gamma>1, \beta=0)$.

The following analogues of Theorems $A$ and $B$ are valid under hypothesis (14). The proofs following [3] are outlined in the appendix.

Theorem C. - Inequality (11) has no solution $y$ which eventually becomes positive as $r \rightarrow \infty$ if $a, b$, and $\varphi$ satisfy (12), (14), and the following:

$$
\begin{aligned}
& \text { (i) } \int_{e}^{\infty} \frac{d r}{a(r)}=\infty \text {; and } \\
& \text { (ii) } \int_{c}^{\infty}\left(\int_{c}^{r} \frac{d s}{a(s)}\right)^{e} b(r) d r=\infty
\end{aligned}
$$

for some $\varrho<1$ and some $e \geqslant 0$.

Theorem D. - Inequality (11) has no solution $y$ which eventually becomes positive as $r \rightarrow \infty$ if $a, b$, and $\varphi$ satisfy (12), (14), and the following:

$$
\begin{aligned}
& \text { (i) } \int_{c}^{\infty} \frac{d r}{a(r)}<\infty ; \\
& \text { (ii) } \int_{c}^{\infty}\left(\int_{r}^{\infty} \frac{d s}{a(s)}\right)^{\mu+\gamma} b(r) d r=\infty
\end{aligned}
$$

for some numbers $\mu>0$ and $c \geqslant 0$.

Theorey 3. - The differential operator $L$ is $h$-ose in an exterior domain $G$ of $E^{n}$ if (2) and (14) hold, and

$$
\int^{\infty} v(r) \lambda(r) d r=\infty
$$


where

$$
\begin{aligned}
v(r) & =r(\log r)^{\varrho} \quad \text { if } n=2 \\
& =r^{\sigma(n)} \quad \text { if } n \geqslant 3, \\
\sigma(n) & =n-1-(\gamma+\mu)(n-2)
\end{aligned}
$$

for some numbers $\varrho<1, \mu>0$, respectively.

Proof, - For $n=2$ we apply Theorem $\mathrm{C}$ in the case that $a(r)=r$ and $b(r)=$ $=r \lambda(r)$ in (11). Hypothesis (ii) of Theorem $\mathrm{C}$ is equivalent to (15) in this case. The proof is then completed in the same way as that of Theorem 1.

For $n \geqslant 3$ we apply Theorem $\mathrm{D}$ in the case that $a(r)=r^{n-1}, b(r)=r^{n-1} \lambda(r)$ in (11), and again use the same argument.

We remark in conclusion that it is a very simple matter to write analogues of Theorems 1-3 when (1) is replaced by

$$
L v=\Delta v+B(x, v, \nabla v) v=0, \quad x \in G
$$

where $B$ is an $m \times m$ real symmetric matrix function with entries of class $C(G \times$ $\left.\times E^{m} \times E^{m}\right)$ such that

$$
[B(x, v, \nabla v) v(x), h] \geqslant \lambda_{h}(|x|) \psi([v(x), h])
$$

whenever $[v(x), h]>0,|h|=1$, under assumptions similar to (2). There are other obvious generalizations, but it is not clear what to do when the operator $\Delta$ is replaced by a more general elliptic operator, or when the exterior domain $G$ is replaced by a more general unbounded domain. In fact, if $G$ is contained in a cone in $E^{2}$ with vertex angle $\alpha<2 \pi$, easy counterexamples similar to those in [3] show that the criterion (15), for instance, is not strong enough for all solutions of (1) to be $h$-ose in $G$.

\section{Appendix.}

Proof of Theorem A. - Suppose to the contrary that there exists a solution $y$ of (11) with $y(r)>0$ throughout $\left[r_{0}, \infty\right)$ for some $r_{0}$. We reduce (11) to the standard form

$$
h^{\prime \prime}(s)+H(s) \varphi[h(s)] \leqslant 0, \quad s \geqslant 0
$$

by the transformations

$$
\begin{gathered}
\alpha(r)=\int_{r_{0}}^{r} \frac{d s}{a(s)}, \quad h(s)=y(\beta(s)), \\
H(s)=a(\beta(s)) b(\beta(s)),
\end{gathered}
$$


where $\beta$ denotes the uniquely determined inverse of the strictly increasing function $\alpha$. Since $h^{\prime \prime}(s) \leqslant 0$ and $h(s)>0$ for $s>0$, it follows routinely that $h^{\prime}(s)>0$ for sufficiently large $s$, say for $s>s_{1}$. Define

$$
g(s)=\frac{s h^{\prime}(s)}{\varphi[h(s)]}, \quad s \geqslant s_{1} .
$$

Then

$$
g^{\prime}(s) \leqslant-s H(s)+\frac{h^{\prime}(s)}{\varphi[h(s)]}
$$

and integration yields

$$
g(s)-g\left(s_{1}\right) \leqslant-\int_{s_{1}}^{s} t H(t) d t+\int_{h\left(s_{1}\right)}^{h(s)} \frac{d h}{\varphi(h)} .
$$

The transformation $t=\alpha(r), r=\beta(t)$ reduces the first integral on the right side to

$$
\int_{s_{1}}^{s} t H(t) d t=\int_{\beta\left(s_{1}\right)}^{\beta(s)} \alpha(r) b(r) d r,
$$

which diverges as $s \rightarrow \infty$ by hypothesis (ii). Hence $g(s)<0$ for sufficiently large $s$ by hypothesis (iii), and consequently $h^{\prime}(s)$ eventually becomes negative. This contradiction completes the proof of Theorem A.

Proof of Theorfim B. - The inequality (11) is reduced to the form

$$
h^{\prime \prime}(s)+H(s) \varphi_{0}[h(s)] \leqslant 0, \quad s \geqslant 0
$$

by the transformations

$$
\begin{gathered}
\alpha(r)=\left(\int_{r}^{\infty} \frac{d s}{a(s)}\right)^{-1}, \quad h(s)=s y(\beta(s)), \\
H(s)=\frac{1}{s^{3}} p_{0}\left(\frac{1}{s}\right) a(\beta(s)) b(\beta(s)),
\end{gathered}
$$

where $\beta$ denotes the inverse function of $\alpha$. The proof is completed in the same way as that of Theorem $\mathbf{A}$ when (18) is replaced by

$$
\int_{s_{1}}^{s} t H(t) d t=\int_{\beta\left(s_{1}\right)}^{\beta(s)} \varphi_{0}\left(\frac{1}{\alpha(r)}\right) b(r) d r,
$$

which diverges by hypothesis (ii) as $s \rightarrow \infty$. 
Proof of Theorem O. - The proof is virtually the same as that of Theorem A, with (16), (17), and (18) replaced by

$$
\begin{gathered}
g(s)=\frac{s^{e} h^{\prime}(s)}{\varphi[h(s)]}, \\
g(s)-g\left(s_{1}\right) \leqslant-\int_{s_{1}}^{s} t e H(t) d t+K \int_{s_{1}}^{s} t^{\varrho-2} d t, \\
\int_{s_{1}}^{s} t e H(t) d t=\int_{\beta\left(s_{1}\right)}^{\beta(s)} \alpha^{\varrho}(r) b(r) d r,
\end{gathered}
$$

respectively, where $K$ is a constant (depending on $\gamma$ and $\varrho$ ). The contradiction $h^{\prime}(s)<0$ for sufficiently large $s$ is then obtained as in the proof of Theorem A.

Proof of Theorem D. - As before, we suppose to the contrary that $y$ is a solution of (11) with $y(r)>0$ throughout $\left[r_{0}, \infty\right)$ for some number $r_{0}$. With $\alpha, \beta$, and $h$ defined the same way as in Theorem $\mathrm{B}$, it follows that there exist numbers $\delta$ and $s_{1}$ such that $y(\beta(s))=s^{-1} h(s) \geqslant \delta$ for all $s \geqslant s_{1}$. Assumption (14) then implies that

$$
\varphi\left(\frac{h(s)}{s}\right) \geqslant K\left(\frac{h(s)}{s}\right)^{\gamma}, \quad s \geqslant s_{1}
$$

for some constants $K>0$ and $\gamma \geqslant 1$. Since $y$ satisfies (11), an easy calculation shows that $h(s)=s y(\beta(s))$ satisfies the differential inequality

$$
h^{\prime \prime}(s)+H(s)[h(s)]^{\gamma} \leqslant 0 \quad s \geqslant s_{1}
$$

where

$$
H(s)=K s^{-3-\gamma} a(\beta(s)) b(\beta(s))
$$

The proof is then completed as before with $\left(16^{\prime}\right),\left(17^{\prime}\right)$, and $\left(18^{\prime}\right)$ replaced respectively by

$$
\begin{gathered}
g(s)=\frac{s^{1-\mu} h^{\prime}(s)}{[h(s)]^{\gamma}} \\
g(s)-g\left(s_{1}\right) \leqslant-\int_{s_{1}}^{s} t^{1-\mu} H(t) d t+K \int_{s_{1}}^{s} t^{-1-\mu} d t, \\
\int_{s_{1}}^{s} t^{1-\mu} H(t) d t=\int_{\beta\left(s_{1}\right)}^{\beta(s)}\left(\frac{1}{\alpha(r)}\right)^{\mu+\gamma} b(r) d r .
\end{gathered}
$$




\section{REFERENCES}

[1] JU. I. DoMšLAK, On the oscillation of solutions of vector differential equations, Dokl. Akad. Nauk SSSR, 193 (1970), pp. 21-23; Soviet. Math. Dokl., 11 (1970), pp. 839-841.

[2] E. S. NoussaIr - C. A. Swanson, Oscillation theorems for vector differential equations, Utilitas Math., 1 (1972), pp. 97-109.

[3] E. S. NoussatR - C. A. Swawson, Oscillation theory for semilinear Sehrödinger equations and inequalities, Proc. Roy. Soc. Edinburgh, Sect. A (to appear).

[4] G. O. OKIKIOLU, Aspects of the Theory of Bounded Integral Operators in $L^{p}$-Spaces, Academic Press, London-New York, 1971. 